American Journal of Agricultural and Biological Sciences 5 (3): 286-293, 2010

ISSN 1557-4989

(C) 2010 Science Publications

\title{
Nasal Administration of Quercetin Liposomes Improves Memory Impairment and Neurodegeneration in Animal Model of Alzheimer's Disease
}

\author{
${ }^{1}$ Terdthai Tong-un, ${ }^{1}$ Supaporn Muchimapura, \\ ${ }^{1}$ Jintanaporn Wattanathorn and ${ }^{2}$ Wathita Phachonpai \\ ${ }^{1}$ Department of Physiology, Faculty of Medicine, \\ ${ }^{2}$ Department of Physiology (Neuroscience Program), Faculty of Medicine, Graduate School, \\ Khon Kaen University, Khon Kaen, Thailand, 40002
}

\begin{abstract}
Problem statement: At present, the development of protective strategy against Alzheimer's Disease (AD) is increasing its importance due to the high prevalence of $\mathrm{AD}$, a limitation of therapeutic efficacy and its high impacts on economic and social aspects. The development of the preventive and therapeutic strategy to protect against the path physiology induced by free radicals in AD from antioxidant has gained very much concentration. Quercetin, one of the flavonoids in fruits and vegetables, has a powerful antioxidant activity both in vitro and in vivo. However, poor absorption, rapid metabolism and limited ability to cross the blood-brain-barrier are obstacles to its use for treatment of AD. Liposome's have been used as an effective delivery system to the brain. Advantages associated with the nasal administration over oral route include higher bioavailability due to no first pass hepatic metabolism and rapid absorption leading to shorter time to onset of effect. Based on all these points, the possible effects of quercetin liposomes via nasal route on improving cognitive behavior and neurodegeneration in animal model of Alzheimer's disease were investigated. Approach: Male Wistar rats were pretreated with quercetin liposome's, containing $0.5 \mathrm{mg}$ of quercetin in $20 \mu \mathrm{L}$ (dose $=20 \mu \mathrm{g}$ ), via intranasal route once daily continually for 2 weeks before and 1 week after AF64A administration. Learning and memory was evaluated using the Morris water maze test at 7 days after the AF64A administration and then the rats were sacrificed for determining the density of neurons and cholinergic neurons in hippocampus using histological and immunohistochemical techniques. Results: Nasal administration of quercetin liposome's significantly prevented changes of spatial memory of AF64A treated rats. The cognitive enhancement of quercetin liposome's was found to be related to its ability to inhibit the degeneration of neurons and cholinergic neurons in hippocampus. Conclusion: Nasal administration of quercetin liposome's possessed cognitive enhancer and neuroprotectant in animal model of $\mathrm{AD}$, suggesting a potential novel therapeutic strategy against $\mathrm{AD}$ of quercetin liposomes.
\end{abstract}

Key words: Quercetin liposome's, nasal administration, Morris water maze test, Alzheimer's disease, spatial memory

\section{INTRODUCTION}

Alzheimer's Disease (AD) is a progressive and complex neurodegenerative disease, characterized by progressive decline in memory, language and other cognitive functions. It is associated with impairment of the basal forebrain cholinergic system, especially in the elderly (Auld et al., 2002). At present, the etiology of $\mathrm{AD}$ is remains a mystery, which makes the treatment of $\mathrm{AD}$ a challenge. Based on the results of extensive experimental work, free radicals appear to be a major culprit causing cellular damage. Several studies provide evidence to link neuronal damage with excessive generation of free radicals, which may be due to factors such as oxidative stress (Olanow, 1993), inflammation (Stuchbury and Munch, 2005), abnormal proteins (Mandelkow et al., 2007) or unknown factors. It has been observed that the use of antioxidants as well as dietary improvements with regard to the consumption of fruits and vegetables high in antioxidant activity and neuroprotective agents may decrease the risk of memory deficits of the AD type (Weinstock and Shoham, 2004).

Quercetin, a member of the flavonoids family, is one of the most prominent dietary antioxidants. It is

Corresponding Author: Terdthai Tong-un, Department of Physiology, Faculty of Medicine, Khon Kaen University, Khon Kaen, Thailand, 40002 Tel: 66-43-348394 
ubiquitously present in foods including vegetables, fruit, tea and wine (Formica and Regelson, 1995), as well as countless food supplements and is claimed to exert beneficial health effects such as antivirus (Ohnishi and Bannai, 1993), certain forms of cancer (Lamson and Brignall, 2000), pulmonary and cardiovascular diseases (Middleton et al., 2000) but also as neroprotection (Cho et al., 2006). The idea of applying quercetin as the agent to improve memory deficit and neuroprotectant has become attractive. In order to apply quercetin for this case, it is necessary to overcome the limitations of quercetin: Poor absorption and very low distribution to the brain after oral administration (Boer et al., 2005) due to both rapid metabolism (Manach et al., 1998) and difficulties in the penetration through the Blood-Brain Barrier (BBB) (Youdim et al., 2004). An effective dose of quercetin to exert certain neurological activities could be relatively high because of first-pass metabolism (Bhattaram et al., 2002).

Liposomes have long been used as a Drug Delivery System (DDS) to the brain, because the particles can entrap the compounds and prevent rapid elimination or degradation as well as promote penetration through the BBB which in turn decrease the effective dose (Sharma and Sharma, 1997). In addition, they do not elicit negative biological responses that generally occur when a foreign material is introduced in the system. These lipid vesicles are non-toxic, non-immunogenic, no carcinogenic, non-thrombogenic and biodegradable (Sinha et al., 2001).

Nasal administration, a noninvasive delivery system, could produce higher bioavailability than oral route due to the decrease hepatic metabolism, shorter distance to brain target and easy penetration through the brain (Wang et al., 2006; Illum, 2000).

Based on this information, we hypothesized that quercetin liposomes via nasal administration could mitigate the memory impairment and neurodegeneration in animal model of Alzheimer's disease.

\section{MATERIALS AND METHODS}

High-purity egg L- $\alpha$-phosphatidylcholine, type XVIE (EPC), cholesterol (chol), quercetin dihydrate (98\% HPLC purity) and Polyethylene Glycol 400 (PEG) were purchased from Sigma (Barcelona, Spain). Other reagents used were analytical grade such as chloroform, ethanol and methanol (HPLC and analytical grade) from BDH Laboratory Supplies (Poole, England), disodium hydrogen phosphate, perchloric acid and ortho phosphoric acids (Merck, Darmstadt, Germany), sodium dihydrogen phosphate (JT Baker Inc., Phillipsburg, New Jersey). All other chemicals were at least reagent grade and used as received.

Preparation of quercetin liposomes: Quercetin dehydrate (98\%), high-purity egg L- $\alpha$ phosphatidylcholine, Type XVI-E (EPC) and Cholesterol (CHOL) were prepared as quercetin liposomes. The method used was lipid thin film formation and extrusion (Guo et al., 2003; Liang et al., 2005; Zu et al., 2006).

Animals: Adult male Wistar rats $(180 \pm 20 \mathrm{~g}, 8$ weeks old) were obtained from National Animal Center, Salaya, Nakhon Pathomand they were housed in group of five per cage in standard metal cages at $22 \pm 2^{\circ} \mathrm{C}$ on 12:12 h light-dark cycle. All animals were given access to food and water ad libitum. The experiments were performed to minimize animal suffering in accordance with the internationally accepted principles for laboratory use and care of European Community (EEC directive of 1986; 86/609/EEC).

The experimental protocols were approved by the Institutional Animal Care and Use Committee.

Experimental protocol: All rats were randomly assigned to five groups of eight animals each:

- Free liposomes + ACSF: the rats in this group were administered free liposomes via nasal route then they were administered artificial cerebrospinal fluid or ACSF bilaterally via intracerebroventricular route

- PEG + ACSF: the rats in this group were administered PEG which used as vehicle of quercetin liposomes via nasal route and subjected to ACSF administration

- Free liposomes + AF64A: all rats were administered free liposomes via nasal route then, they were administered AF64A bilaterally via intracerebroventricular route

- PEG + AF64A: the animals were administered PEG via nasal route then they were administered AF64A as mentioned in group 3

- $\mathrm{QCL}+\mathrm{AF} 64 \mathrm{~A}$ : the rats in this group were administered quercetin liposomes via nasal route, then they were administered AF64A

All animals were administered freshly prepared quercetin liposomes, containing $0.5 \mathrm{mg}$ of quercetin in $20 \mu \mathrm{L}($ dose $=20 \mu \mathrm{g})$, or liposomes without quercetin at the same volume via right nasal cavity with a micropipette at a period of 2 weeks before and 1 week 
after AF64A or Artificial Cerebrospinal Fluid (ACSF) administration. The animals were determined the spatial memory 1 week after AF64A administration then they were sacrificed and determined the density of survival neurons and cholinergic neurons in various subregions of hippocampus.

AF64A administration: AF64A was prepared as described previously by Hanin (1996). Briefly, an aqueous solution of acetylethylcholine mustard $\mathrm{HCl}$ (Sigma, St. Louis, MO) was adjusted to $\mathrm{pH} 11.3$ with $\mathrm{NaOH}$. After stirring for $30 \mathrm{~min}$ at room temperature, the $\mathrm{pH}$ was lowered to 7.4 with the gradual addition of $\mathrm{HCl}$ and stirred for $60 \mathrm{~min}$. The amount of AF64A was then adjusted either to $2 \mathrm{nmol} / 2 \mu \mathrm{L}$. The vehicle of AF64A was distilled water prepared in the same manner as the AF64A and recognized as ACSF. In order to administer AF64A bilaterally via intracerebroventricular (i.c.v.) route, the animals were anesthetized with the intraperitoneal injection of sodium pentobarbital at dose of $60 \mathrm{mg} \mathrm{kg}^{-1} \mathrm{BW}$. Then, AF64A $(2 \mathrm{nmol} / 2 \mu \mathrm{l})$ was infused bilaterally via intracerebroventricular (i.c.v.) route with a 30-gauge needle inserted through a burr hole drilled into the skull into both the right and left lateral ventricles. Stereotaxic coordinates were (from the bregma): Posterior $0.8 \mathrm{~mm}$, lateral $\pm 1.5 \mathrm{~mm}$ and ventral (from dura) $3.6 \mathrm{~mm}$. The rate of infusion was $1.0 \mu \mathrm{min}^{-1}$ and the needle was left in place for $5 \mathrm{~min}$ after infusion and then slowly withdrawn.

Morris water maze test (behaviors evaluation): The water maze consisted of a metal pool $(170 \mathrm{~cm}$ in diameter $\times 58 \mathrm{~cm}$ tall $)$ filled with tap water $\left(25^{\circ} \mathrm{C}\right.$, $40 \mathrm{~cm}$ deep) divided into four quadrants. In the center of one quadrant was a removable escape platform below the water level and covered with a nontoxic milk powder. The pool was divided into four quadrants (NE, NW, SE and SW) by two imaginary lines crossing the center of the pool. For each animal, the location of invisible platform was placed at the center of one quadrant and remained there throughout training. The rats must memorize the platform location in relation to various environmental cues because there was nothing directly shows the location of the escape platform in and outside the pool. Therefore, the placement of the water tank and platform were the same in all acquisition trials. Each rat was gently placed in the water facing the wall of the pool from one of the four starting points $(\mathrm{N}$, $\mathrm{E}, \mathrm{S}$, or $\mathrm{W}$ ) along the perimeter of the pool and the animal was allowed to swim until it found and climbed onto the platform. During training session, the subject was gently placed on the platform by the experimenter when it could not reach the platform in 60 sec. In either case, the subject was left on the platform for $15 \mathrm{sec}$ and removed from the pool. The time for animals to climb on the hidden platform was recorded as escape latency or acquisition time. In addition to the acquisition test, the determination of retention memory was carried out on the next day. According to this test, the platform was removed and the animals were placed into the water maze for $60 \mathrm{sec}$. The retention of memory or the time that the animal spent to swim around the previous location of platform before removing the platform on the test occurring in the next day was also recorded. It has been postulated that if the spatial memory of the rat for the trained platform location is accurate, the rat will swim to the platform location and search around the exact location. Therefore, the more accurate the spatial memory is, the greater the number of times rat swim across the trained platform. In each trial, the animal was quickly dried with towel before being returned to the cage. All test in Morris water maze tests were carried out within $30 \mathrm{~min}$ after the nasal administration of the substances.

Any enhancement of cognition would be reflected by a decrease in escape latency and increase in retention time.

Histological procedure: Following anesthesia with sodium pentobarbital $\left(60 \mathrm{mg} \mathrm{kg}^{-1} \mathrm{BW}\right)$, fixation of the brain was carried out by transcardial perfusion with fixative solution containing $4 \%$ par formaldehyde in $0.1 \mathrm{M}$ phosphate buffer $\mathrm{pH}$ 7.3. The brains were removed after perfusion and stored over a night in a fixative solution that used for perfusion. Then, they were infiltrated with $30 \%$ sucrose solution for approximately $4^{\circ} \mathrm{C}$. The specimens were frozen rapidly and $30 \mu \mathrm{M}$ thick sections were cut on cryostat. They were rinsed in the phosphate buffer and picked up on slides coated with $0.01 \%$ of aqueous solution of a high molecular weight poly L-lysine.

Nissl staining: The duplicate coronal sections of brains were stained with $0.75 \%$ cresyl violet, dehydrated through graded alcohols $(70,95,100 \% 2 \times)$, placed in xylene and coverslipped using DPX mountant.

Choline acetyltransferase and immunohistochemistry: A series of section containing hippocampus from each groups were reacted in parallel experiments using a mouse monoclonal antibody detected against Choline Acetyltransferase (ChAT) (Chemicon Internation, Inc., CA, USA) and a modification of a previously described protocol employing the DAKO Strept ABC Complex/HRP duet 
kit. In brief, the sections were eliminated endogenous peroxidase activity by $0.5 \% \mathrm{H} 2 \mathrm{O} 2$ in methanol. Sections were washed in running tap water and distilled water for 1 min each, then rinsed in KPBS and KPBS-BT for 5 min per each process. Excess was removed, then incubated for $30 \mathrm{~min}$ in a blocking solution composed of 5\% normal horse serum in KPBS-BT. The sections were then incubated in mouse primary antibody against ChAT diluted $1: 100$ in KPBS-BT at room temperature for $2 \mathrm{~h}$ and then incubated at $4 \mathrm{C}$ for $48 \mathrm{~h}$. The tissue was rinsed in KPBS-BT (two washes $\times 7$ min), incubated for $4 \mathrm{~h}$ in biotinylated goat antimouse IgG antibody, rinsed in KPBS-BT (two washes $\times 7 \mathrm{~min}$ ) and then incubated in Strep ABCComplex/HRP for $4 \mathrm{~h}$. In preparation for visualization step, sections were rinsed in KPBS-BT ( $1 \mathrm{~min}$ ) and KPBS (two washes $\times 10 \mathrm{~min}$ ). ChAT immunoreactivity was visualized using $0.025 \%$ 3, 3' Diaminobenzidine (DAB, Sigma) and $0.01 \%$ $\mathrm{H}^{2} \mathrm{O}^{2}$. Finally, sections were rinsed in running tap water, air dried and cover-slipped using permount.

Statistical analysis: Data are presented as mean \pm standard error of mean ( \pm SEM). One-way Analysis Of Variance (ANOVA), followed by Duncan's post hoc test. A probability level less than 0.05 was accepted as significance.

\section{RESULTS}

Effect of quercetin liposomes on spatial memory in AF64A treated rats: Figure 1 and 2 shows that ACSF produced no significant changes on both escape latency and retention time in Morris water maze test. Intracerebroventricular administration of AF64A significantly increased escape latency but decreased retention time ( $\mathrm{p}$-value $<0.001$ all; compared to both liposomes + ACSF and PEG + ACSF). This indicated the memory impairment induced by AF64A. Rats that received AF64A and nasal administration of quercetin liposomes showed significantly decreased acquisition and increased retention time (p-value $<0.001$ all; compared to liposomes + ACSF and PEG + AF64A). Therefore, our data indicated that quercetin liposomes via nasal administration improved the memory deficit induced by AF64A.

Neuroprotective effect of quercetin liposomes in AF64A treated rats: The effect of quercetin liposomes on the neurons density in various subregions of hippocampus was shown in Fig. 3. Both liposomes and PEG had been previously demonstrated to produce no harmful effect on neuron density. The administration of
AF64A in either liposomes or PEG treated groups significantly decreased the neuron density in CA1, CA2, CA3 and dentate gyrus (p-value $<0.001$ all; compared to both liposomes + ACSF and PEG + ACSF). Surprisingly, quercetin liposomes administration could reverse these changes (p-value0.001 all; compared to liposomes + ACSF and PEG + AF64A).

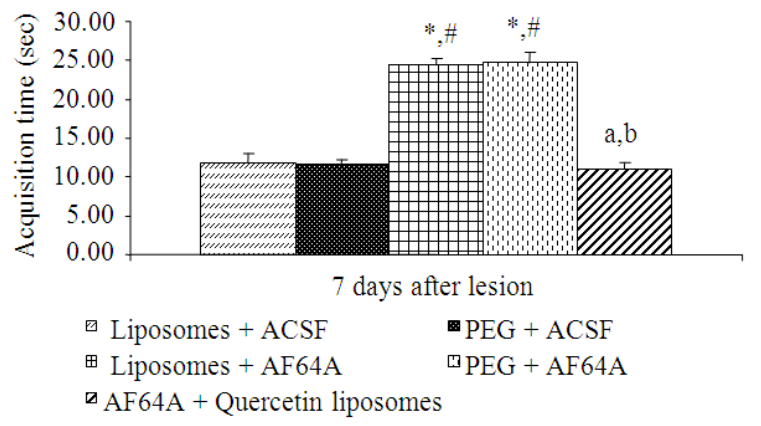

Fig. 1: Effect nasal administration of quercetin liposomes on the acquisition time of spatial learning in the Morris water maze test $(\mathrm{N}=8)$. Results were expressed as mean $\pm \mathrm{SEM}$; ${ }^{*}$ : Pvalue $<0.001$ compared with liposomes + ACSF treated group; \#: p-value $<0.001$ compared with PEG + ACSF treated group; ${ }^{a}: p$-value $<0.001$ compared with liposomes + AF64A treated group; ${ }^{\text {b }}$ : p-value $<0.001$ compared with PEG + AF64A treated group

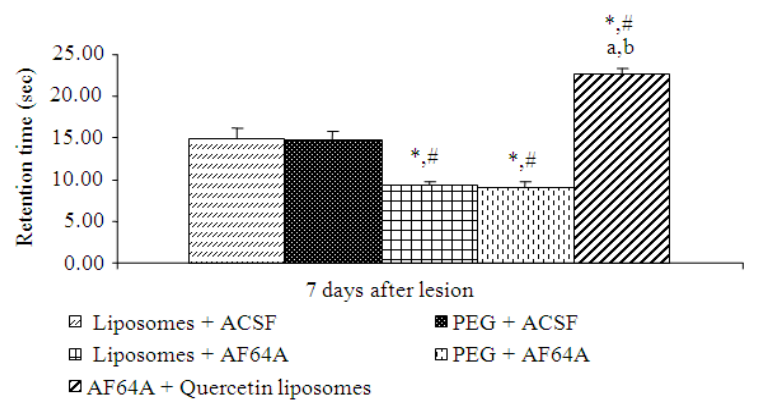

Fig. 2: Effect nasal administration of quercetin liposomes on the retention time of spatial learning in the Morris water maze test $(\mathrm{N}=8)$. Results are expressed as mean $\pm \mathrm{SEM}$; ${ }^{*}$ : pvalue $<0.001$ compared with liposomes + ACSF treated grou; \#: p-value $<0.001$ compared with PEG + ACSF treated group; ${ }^{a}: \mathrm{p}$-value $<0.001$ compared with liposomes + AF64A treated group; ${ }^{\text {b }}$ : p-value $<0.001$ compared with PEG + AF64A treated group 


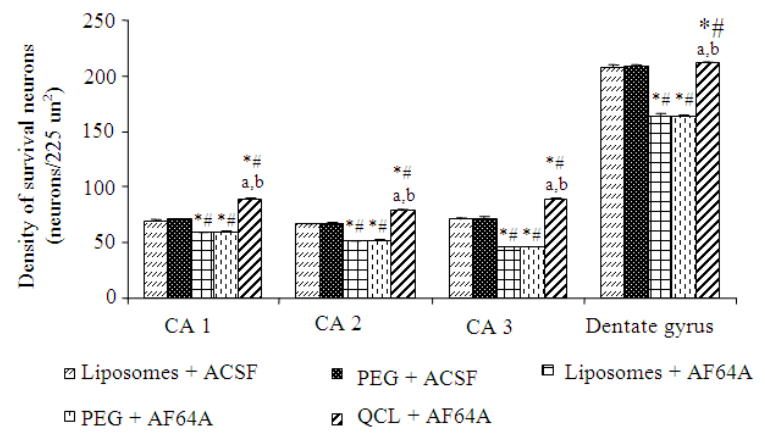

Fig. 3: Effect nasal administration of quercetin liposomes on the density of neurons in hippocampus. $(\mathrm{N}=8)$ Results were expressed as mean \pm SEM; *: p-value $<0.001$ compared with liposomes + ACSF treated group; \#: p-value<0.001 compared with PEG + ACSF treated group; ${ }^{\mathrm{a}}$ : p-value $<0.001$ compared with liposomes + AF64A treated group; b: p-value<0.001 compared with PEG + AF64A treated group

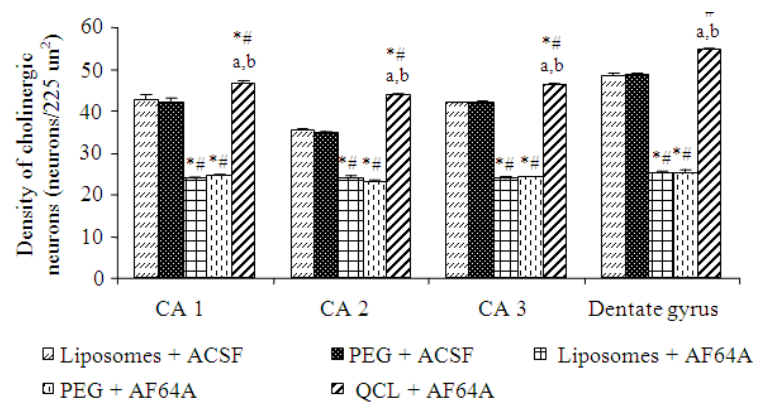

Fig. 4: Effect nasal administration of quercetin liposomes on the density of cholinergic neurons in hippocampus. Results are expressed as mean \pm SEM; ${ }^{*}$ : p-value $<0.001$ compared with liposomes + ACSF treated group; \#: p-value<0.001 compared with PEG + ACSF treated group; ${ }^{\mathrm{a}}: \mathrm{p}$-value $<0.001$ compared with liposomes + AF64A treated group; b: p-value $<0.001$ compared with PEG + AF64A treated group

We also determined the effect of all interventions mentioned earlier on the density of cholinergic neurons in hippocampus, the area playing an important role on spatial memory. Data were shown in Fig. 4. AF64A produced significant reduction in cholinergic neurons densities in all areas of hippocampus (p-value $<0.001$ all; compared to both liposomes + ACSF and PEG +
ACSF) whereas quercetin liposomes could significantly attenuate the decrease cholinergic neurons densities induced by AF64A in all subregions of hippocampus (p-value<0.001 all; compared to liposomes + AF64A and PEG + AF64A).

\section{DISCUSSION}

The current study demonstrates that nasal administration of quercetin liposomes could mitigate the memory impairment and the degeneration of neurons and cholinergic neurons in hippocampus in animal model of Alzheimer's disease induced by AF64A.

Various degrees of cognitive impairment were reported to be associated with cholinergic neuronal loss and dysfunction especially in AD (Bondy, 1995). Recently, a pile of evidence suggested that the prime candidate responsible for producing the neuronal changes mediating these cognitive deficits appeared to be free radicals and oxidative stress generated (Mattson, 2004; Cantuti et al., 2000). Therefore, many studies focused on the beneficial effects of supplement possessing a capability to improve cholinergic function and antioxidant activity were also considered to be a potential candidate for neuroprotective agent against AD.

Quercetin, a main flavonoid found in fruits, vegetables and beverages, was reported to possess antioxidant and cognition (Boots et al., 2008; Reiter et al., 2009). Previous studies confirm that quercetin supplementation improve memory deficit condition induced by reserpine in mice (Naumenko and Kulikov, 2006). However, it was reported that quercetin was easily metabolized after absorption (Manach et al., 2004). Our study found it worthwhile to use the vesicle mediated carrier system particularly liposomes to encapsulated the quercetin against Alzheimer's condition induced by AF64A.

Intracerebroventricular injection by AF64A has been described as an appropriate the substance to destroy the cholinergic system and induce memory deficit (Hanin, 1996; McDonald and Overmier, 1998). In the present study, the results show that both in the AF64A + free liposomes and AF64A + PEG treated groups, caused a persistent memory deficit as evidenced by a significant induction in acquisition time and reduction in retention time in Morris water maze. This deficit in memory was reversed by quercetin liposomes via nasal route. Pretreatment with nasal administration of quercetin liposomes improved spatial memory as evidenced by improved performance in Morris water maze test. 
The evidence stems from data of several authors demonstrated that memory impairment especially the spatial memory or hippocampal dependent memory was tightly associated with the neurodegeneration in hippocampus, which in turn depended on the density of neurons particularly cholinergic neurons (Katzman, 1986; Mori et al., 1997). Our results demonstrated that in the AF64A + free liposomes and AF64A + PEG treated groups, there were the reduction of survival neurons and cholinergic neurons in all areas of hippocampus. Interestingly, in AF64A with nasal administration of quercetin liposomes treated group, the rats showed significantly improved the density of survival neurons and cholinergic neurons in all areas of hippocampus as compared to the AF64A + PEG or free liposomes treated rats. Moreover, it was notable that the density of cholinergic neurons of quercetin liposomes plus AF64A treated group were higher than those obtained from the liposomes + ACSF and PEG + ACSF treated group. This suggests that quercetin liposomes not only the neuroprotective effect but also the neurotrophic effect to stimulate the neurogenesis in hippocampus. Previous study demonstrated that the neurogenesis could occur throughout adulthood especially in hippocampus and subventricular zone of lateral ventricle (Hagg, 2005). Numerous factors had been reported to be regulators of the adult neurogenesis. To clarify this issue, further researches are still essential.

Our results demonstrated that nasal administration of quercetin encapsulated liposomes was a potential novel strategy to protect against neurodegeneration such as AD. It was noticeable that the dose of quercetin required via nasal administration was very much low dose and rapid onset of cognitive enhancing effect. In this respect, quercetin could be delivered to the brain even though it was administered in nondissolved form in a liquid that was not a good candidate for delivery of this hydrophobic compound (Cho et al., 2006). Some of the suspended particles of quercetin might not be absorbed after all. Our present study suggests that the enhanced delivery of quercetin in the form of liposomes to the brain could effectively reduce the dose, which would also reduce the potential of toxicity of the substance (Metodiewa et al., 1999) and increase bioavailability (Jamal et al., 2000). Moreover, it was also provided numerous benefits especially the direct nose-to-brain delivery, bypassing the blood brain barrier (Wu et al., 2008) and avoid the first pass metabolism (Graf et al., 2006). However, the main proposed pathway of quercetin delivery via nasal administration was different. It was likely to permeate through the subarachnoid space through the olfactory epithelium and found in the CSF later, because the liposomes behaved as semilipophilic particles. Therefore, quercetin liposomes could rapidly absorb into the CSF. Investigation of quercetin absorption and distribution after administered via nasal route would be of value for future studies.

\section{CONCLUSION}

Nasal administration of quercetin liposomes improves both memory impairment and neurodegeneration in cognitive deficit condition induced by AF64A, suggesting a potential valuable candidate for cognitive enhancer and neuroprotective agent in $\mathrm{AD}$.

\section{ACKNOWLEDGEMENT}

This study was partial supported by the National Nanotechnology Center (NANOTEC), NSTDA, National Science and Technology Development Agency, Thailand, through its program of Center of Excellence Network.

\section{REFERENCES}

Auld, D.S., T.J. Kornecook, S. Bastianetto and R. Quirion, 2002. Alzheimer's disease and the basal forebrain cholinergic system: Relations to $\beta$ amyloid peptides cognition and treatment strategies. Prog. Neurobiol., 68: 209-245. PMID: 12450488

Bhattaram, V.A., U. Graefe, C. Kohlert, M. Veit and H. Derendorf, 2002. Pharmacokinetics and bioavailability of herbal medicinal products. Phytomedical, 9: 1-33. DOI: 10.1078/1433-187X00210

Boer, V.C.J., A.A. Dihal, H. Van Der Woude, I.C.W. Arts and S. Wolffram et al., 2005. Tissue distribution of quercetin in rats and pigs. J. Nutr., 135:1617-1618. PMID: 15987855

Bondy, S.C., 1995. The relation of oxidative stress and hyperexcitation to neurological disease, Proc. Soc. Exp. Biol. Med., 208: 337-345. PMID: 7700883

Boots, A.W., G.R. Haenen and A. Bast, 2008. Health effects of quercetin: From antioxidant to nutraceutical. Eur. J. Pharmacol., 585: 325-337. DOI: 10.1016/j.ejphar.2008.03.008

Cantuti, C.I., B. Shukitt-Hale and J.A. Joseph, 2000. Neurobehavioural aspects of antioxidants in aging. J. Neurosci., 18: 367-381. DOI: 10.1016/S07365748(00)00008-3 
Cho, J.Y., I.S. Kim, Y.H. Jang, A.R. Kim and S.R. Lee, 2006. Protective effect of quercetin, a natural flavonoid against neuronal damage after transient global cerebral ischemia. Neurosci. Lett., 404: 330-335. PMID: 16806698 DOI: 10.1016/j.neulet.2006.06.010

Formica, J.V. and W. Regelson, 1995. Review of the biology of quercetin and related bioflavonoids. Food. Chem. Toxicol., 33: 1061-1080. PMID: 8847003

Graf, B.A., C. Ameho, G.G. Dolnikowski, P.E. Milbury and C.Y. Chen et al., 2006. Rat gastrointestinal tissues metabolized quercetin. J. Nutr., 136: 39-44. PMID: 16365056

Guo, J., Q. Ping, G. Jiang, L. Huang and Y. Tong, 2003. Chitosan-coated liposomes: Characterization and interaction with leuprolide. Int. J. Pharm., 260: $\quad 167-173 . \quad$ DOI: $\quad 10.1016 / S 0378-$ 5173(03)00254-0

Hagg, T., 2005. Molecular regulation of adult CNS neurogenesis: An integrated view. Trends Neurosci., 28: 589-595. DOI: 10.1016/j.tins.2005.08.009

Hanin, I., 1996. The AF64A model of cholinergic hypofunction: An update. Life Sci., 58: 1955-1964. DOI: 10.1016/0024-3205(96)00185-3

Illum, L., 2000 Transport of drugs from the nasal cavity to the central nervous system. Eur. J. Pharm. Sci., 11: 1-18. PMID: 10913748

Jamal, T., S. Jean-Michel, R.R. Anthony and K. Michel, 2000. Brain drug delivery technologies: Novel approaches for transporting therapeutics. Pharm. Sci. Technol., 3: 155-162. PMID: 10785657

Katzman, R., 1986. Alzheimer's disease. N. Engl. J. Med., 314: 964-973. PMID: 2870433

Lamson, D.W. and M.S. Brignall, 2000. Antioxidants and cancer. Part III. quercetin. Altern. Med., 5: 196-208. PMID: 10869101

Liang, M.T., N.M. Davies and I. Toth, 2005. Encapsulation of lipopeptides within liposomes: Effect of number of lipid chains, chain length and method of liposome preparation. Int. J. Pharm., 301: 247-254. PMID: 16054787 DOI: 10.1016/j.ijpharm.2005.06.010

Manach, C., C. Morand, V. Crespy, C. Demignee and O. Texier et al., 1998. Quercetin is recovered in human plasma as conjugated derivatives which retain antioxidant properties. FEBS Lett., 426: 331-336. PMID: 9600261

Manach, C., A. Scalbert, C. Morand, C. Remesy and L. Jimenez, 2004. Polyphenols: Food sources and bioavailability. Am. J. Clin. Nutr., 79: 727-747. PMID: 15113710
Mandelkow, E., M.V. Bergen, J. Biernet and E.V. Mandelkow, 2007. Structural principles of tau and the paired helical filaments of Alzheimer's disease. Brain. Pathol., 17: 83-90. PMID: 17493042

Mattson, M.P., 2004. Pathways towards and away from Alzheimer's disease. Nature, 430: 631-639. DOI: 10.1038/nature02621

McDonald, M.D. and J.B. Overmier, 1998. Present imperfect: A critical review of animal models of the mnemonic impairments in Alzheimer's disease. Neurosci. Biobehav. Rev., 22: 99-120. DOI: 10.1016/S0149-7634(97)00024-9

Metodiewa, D., A.K. Jaiswal, N. Cenas, E. Dickancaite and J. Segura-Aguilar, 1999. Quercetin may act as a cytotoxic prooxidant after its methanolic activation to semiquinone and quinoidal product. Free. Radic. Biol. Med., 26: 107-116. PMID: 9890646

Middleton, E., C. Kandaswami and T.C. Theoharides, 2000. The effects of plant flavonoids on mammalian cells: implications for inflammation, heart disease and cancer. Pharm. Rev., 52: 673-751. PMID: 11121513

Mori, E., H. Hirono, H. Yamashita, T. Imamura and Y. Ikejiri et al., 1997. Premorbid brain size as a determinant of reserve capacity against intellectual decline in Alzheimer's disease. Am. J. Psychiat., 154: 18-24. PMID: 8988953

Naumenko, V.S. and A.V. Kulikov, 2006. Quantitative assay of 5-HT (1A) serotonin receptor gene expression in the brain. Molekuliarnaia Biol., 40: 37-44. PMID: 16523690

Ohnishi, E. and H. Bannai, 1993. Quercetin potentiates TNF-induced antiviral activity. Antiv. Res., 22: 327-331. PMID: 8279819

Olanow, C.W., 1993. Aradical hypothesis for neurodegeneration. Trends Pharma. Sci., 16: 439. PMID: 7507613

Reiter, M., K. Rupp, P. Baumeister, S. Zieger and U. Harreus, 2009. Antioxidant effects of quercetin and coenzyme Q10 in mini organ cultures of human nasal mucosa cells. Anticancer Res., 29: 33-39. PMID: 19331131

Sharma, A. and U.S. Sharma, 1997. Liposomes in drug delivery: Process and limitations. Int. J. Pharm., 154: 123-140.

Sinha, J., N. Das and M.K. Basu, 2001. Liposomal antioxidants in combating ischemia-reperfusion injury in rat brain. Biomed. Pharmacol. Ther., 55: 264-271. DOI: 10.1016/S0753-3322(01)00060-9

Stuchbury, G. and G. Munch, 2005. Alzheimer's associated inflammation, potential drug targets and future therapies. J. Neural Transm., 112: 429-453. PMID: 15723159 
Wang, X., H. He, W. Leng and X. Tang, 2006. Evaluation of brain-targeting for the nasal delivery of estradiol by the microdialysis method. Int. J. Pharm., $\quad 317$ : 40-46. $\quad$ DOI: 10.1016/j.ijpharm.2006.02.055

Weinstock, M. and S. Shoham, 2004. Rat models of dementia based on reductions in regional glucose metabolism, cerebral blood flow and cytochrome oxidase activity. J. Neural Transm., 111: 347-366. PMID: 14991459

Wu, H., K. Hu and X. Jiang, 2008. From nose to brain: Understanding transport capacity and transport rate of drugs. Expert Op. Drug Deliv., 5: 1159-1168. PMID: 18817519
Youdim, K.A., M.Z. Qaiser, D.J. Begley, C.A. RiceEvans and N.J. Abbott, 2004. Flavonoid permeability across an in situ model of the bloodbrain barrier. Free. Radic. Biol. Med., 36: 592-604. PMID: 14980703

Zu, Y., C. Li, Y. Fu and C. Zhao, 2006. Simultaneous determination of catechin, rutin, quercetin kaempferol and isorhamnetin in the extract of sea buckthorn (Hippophae rhamnoides L.) leaves by RP-HPLC with DAD. J. Pharm. Biomed. Anal., 41: 714-719. PMID: 16520013 